\section{$\underset{\substack{\text { hommes } \\ \text { \& migrations }}}{ }$}

\section{Hommes \& migrations}

Revue française de référence sur les dynamiques

migratoires

1317-1318 | 2017

L'Europe en mouvement

\title{
Documenter la vie quotidienne de gens ordinaires
}

Entretien avec Jacques Windenberger réalisé en novembre 2014

\section{Anne Volery}

\section{(2) OpenEdition}

12 Journals

\section{Édition électronique}

URL : http://journals.openedition.org/hommesmigrations/3922

DOI : 10.4000/hommesmigrations.3922

ISSN : 2262-3353

\section{Éditeur}

Musée national de l'histoire de l'immigration

\section{Édition imprimée}

Date de publication : 1 avril 2017

Pagination : 180-185

ISBN : 978-2-919040-38-4

ISSN : $1142-852 X$

\section{Référence électronique}

Anne Volery, «Documenter la vie quotidienne de gens ordinaires », Hommes \& migrations [En ligne], 1317-1318 | 2017, mis en ligne le 01 avril 2017, consulté le 15 septembre 2020. URL : http:// journals.openedition.org/hommesmigrations/3922 


\section{REPÉRAGE}

Jacques Windenberger, Fos-sur-Mer, sur le chantier de l'usine sidérurgique SOLMER, 9 mai 1972. ( ) MUSÉE NATIONAL DE L'HISTOIRE

DE L'IMMIGRATION, SAIF.

\section{DOCUMENTER LA VIE QUOTIDIENNE DE GENS ORDINAIRES}

Propos recueillis par ANNE VOLERY lors d'un entretien avec JACQUES WINDENBERGER réalisé en novembre 2014.

Jacques Windenberger est photographe. 39 de ses photographies font partie des collections $d u$ Musée national de l'histoire et des cultures de l'immigration. Depuis les années 1960, il a fait le choix de la photographie documentaire et il a construit son travail sur une démarche rigoureuse.
Au cours de ses multiples travaux, il a plusieurs fois photographié des immigrés avec, toujours, une double focale: "documenter », grâce à la photographie, les situations qu'il rencontre et montrer « la vie quotidienne de gens ordinaires". Avec son travail, c'est une photographie ayant 
«"Une civilisation démocratique

ne se sauvera que si elle fait du

langage de l'image une provocation

à la réflexion et non une invite à

l'hypnose." Cette citation d'Umberto

Eco (d'un écrit des années 1950

ou 1960), est encore d'unegrande

actualité en 2014. »

Jacques Windenberger.

un rôle social que nous rencontrons : il attend des images qu'elles "suscitent le débat, qu'elles questionnent le réel " et, en même temps, qu'elles mettent en lumière et laissent des traces. Jacques Windenberger est né en 1935 à Bourgen-Bresse. D'abord rédacteur pour la presse écrite, il rejoint en 1959 l'agence de presse photographique Keystone à Paris. II commence alors une carrière de photo-journaliste. Très vite, il quitte le milieu des agences pour devenir photographe indépendant. Pas de spectaculaire ni de faits divers dans la façon dont il traite le thème de l'immigration. Au contraire, c'est par le biais de la vie quotidienne et du travail, qu'il aborde le sujet. C'est d'ailleurs en traitant des sujets liés à l'industrie, à la sidérurgie, à l'agriculture ou encore à l'urbanisme qu'il s'est retrouvé à travailler sur l'immigration. En tirant le fil du travail, c'est " la vie quotidienne dans sa banalité souvent invisible » qu'il est amené à montrer.

\section{Hommes \& Migrations:}

\section{Pouvez-vous vous présenter?}

Jacques Windenberger : Je suis journaliste. J'ai commencé comme rédacteur dans un petit quotidien où je faisais de la locale à Bourg-en-Bresse, dans l'Ain, où je suis né. Puis j'ai été malade et j'ai fait un centre d'apprentissage réservé à d'anciens malades. Je suis allé ensuite à Paris où, après un bref stage à la revue Réalités, stage que j’ai pu faire grâce à Édouard Boubat, j'ai travaillé durant un an, un an et demi, dans une agence de presse qui s'appelait Keystone. Ensuite, je suis devenu indépendant. Photographe indépendant. Parce que j'en avais assez de prendre des images, selon moi peu intéressantes, de pseudo-événements : des cocktails parisiens, des sorties de l'Élysée, etc. J'habitais à Sarcelles, dans le premier grand ensemble qui a été construit en France, cité où je vivais une vie ordinaire, je dirais vécue par le plus grand nombre. Et il y avait un tel écart entre ce qu'on me faisait faire dans cette agence et la « vie » que je me suis dit qu'il y avait autre chose à faire avec la photographie.

C'est ainsi que j'ai suivi en tant qu'habitant la vie dans cette cité... J'habitais Sarcelles et, en même temps, je chroniquais, je documentais la vie quotidienne telle qu'elle s'y déroulait, sous ses différents aspects. Depuis les débuts, j'entends par "vie quotidienne » les temps, les activités, les besoins de tout un chacun. L'habitat, le travail, les transports, la consommation, les loisirs, la vie sociale, la vie politique, les religions... en essayant de ne pas dissocier ces différents aspects. On n'est pas d'un côté producteur, de l'autre consommateur. Tout cela est lié. Je suis très attaché à la transversalité et aux interdépendances entre les choses. C'est pareil en ce qui concerne l'immigration : il importe de ne pas dissocier ce thème ni de la population en général, ni d'isoler les immigrés en tel ou tel lieu. Je pense que tout se tient et que, quand on a une focale unique, ce sont des œillères qui ne permettent pas de comprendre, d'aborder ou de représenter au mieux la complexité du monde.

Pour revenir à mon parcours, pendant toute la période des années 1960, j’ai travaillé sans commande, c'est-à-dire " en spéculation », comme à Sarcelles, où c'était assez facile puisque j'y résidais. Mais j'ai aussi travaillé "sur commande", notamment dans le milieu rural, puisque chaque mois j'avais un reportage à faire pour le Centre national des jeunes agriculteurs. C'est ainsi que j'ai parcouru pratiquement tous les départements de France pour de courtes incursions. Je dois aussi rappeler, pour donner quelques jalons dans mon travail, qu'en 1962-1963, j'ai été res- 


\section{COLLECTIONS}

ponsable d'une mission d'éducation de base au Rwanda. Ce séjour de quatre à cinq mois dans ce pays a éveillé en moi une conscience politique mondiale puisque je voyais là l'écart qu'il y avait entre la vie des paysans, jeunes ou pas, en France et la condition ordinaire des Rwandais. Cela a été pour moi une révélation qui n'est peut-être pas sans rapport avec mon intérêt, ensuite, pour la condition des immigrés.

\section{H\&M : Comment avez-vous commencé à travailler sur l'immigration ?}

J. W. : L'immigration. Je dirais que je n'ai pas fait exprès, ily a cinquante ans, de m'y intéresser, mais je l'ai rencontrée dans les divers sujets touchant à l'urbanisme, à l'agriculture, à l'industrie que j'ai pu traiter : qu'il s'agisse de la construction d'une cité comme Sarcelles, des conditions de vie des immigrés saisonniers agricoles que j'ai pu rencontrer en région Paca et aussi dans le Languedoc; qu'il s'agisse également de l'industrie ou de la sidérurgie à Fos-sur-Mer et dans l'Est, où j'ai mené un travail issu d'une commande publique du ministère de l'Aménagement du territoire en Lorraine où, là encore, ils étaient présents.

Finalement, je n'ai pas fait ce qu'on fait d'excellents photographes qui ont choisi de montrer des moments forts ou singuliers. Je pense à Olivier Jobard et à son travail sur Kingsley, un immigré clandestin qu'il a suivi dans le bateau jusqu'en France et qu'il a retrouvé après, ou au travail de Patrick Zachmann sur les traversées en bateaux pour aller à Lampedusa ou pour arriver ensuite à Calais. Mon créneau, si je puis m'exprimer ainsi, c'est la vie quotidienne dans sa banalité, qui est souvent invisible, des immigrés en France ou à l'étranger.

$H \& M$ : Les photographies que nous avons en collection au Musée sont issues de deux grands travaux que vous

\section{avez menés : le complexe industriel de Fos-sur-Mer et l'immigration à Marseille. Pouvez-vous nous en parler?}

J. W. : Je suis arrivé à Aix-en-Provence en 1969 à l'occasion de la mise en œuvre du complexe industriel de Fos-sur-Mer. J'ai essayé à ce moment-là de trouver le moyen de travailler de manière régulière sur cet énorme chantier, qui était le plus important au niveau européen et dans lequel étaient employés de nombreux immigrés qui vivaient là dans des conditions plus que précaires. J'ai pu voir que beaucoup étaient logés dans des Algeco plus que sommaires, sans eau. Je les ai photographiés, là aussi, dans leur habitat et dans leur travail sur les chantiers.

À Marseille, j'ai travaillé avec une équipe du CNRS dans le cadre d'une action thématique programmée d'observation du changement social et culturel. Je travaillais non seulement sur le cœur de Marseille, c'est-à-dire le Panier, mais aussi sur les quartiers nord. Après le projet avec le CNRS, j'ai proposé un autre projet, personnel, dans les années 1990, qui a été réalisé dans le cadre de la politique de la ville, avec des cofinancements. Pendant trois ans, j'ai travaillé auprès de trois familles immigrées : une famille comorienne dans le quartier du Panier, une famille andalouse monoparentale dans l'un des quartiers nord, à la Bricarde, et une famille kabyle dans un bidonville, toujours dans les quartiers nord, à la Lorette. C'était un travail passionnant, toujours axé sur la vie réelle. Ce projet, intitulé Question de fraternité, visait à montrer les immigrés non pas comme la presse en parlait le plus souvent, à l'occasion de faits divers. Moi ce qui m'intéressait, c'était de montrer que c'étaient des gens avec une vie qu'ils essayaient de rendre la plus banale possible, en tâchant de résoudre les besoins du quotidien, que ce soit pour se loger ou pour travailler. 
H\&M : Comment traite-t-on de ces sujets en photographie? Comment se construit votre travail ?

J. W. : Là, c'est de la photographie documentaire dont il faut parler. Celle que j'ai essayé d'enseigner pendant dix ans à Aix-en-Provence à la Faculté d'Arts plastiques. Elle exige du temps.

Pour prendre la définition de Life, c'est la description du monde réel par un photographe dont l'intention - il y a bien sûr une subjectivité - est de communiquer quelque chose d'important, c'est-à-dire de faire un commentaire qui soit compris par le destinataire.

Il faut trouver, détecter, des sujets ou des circonstances qui paraissent importants pour le photographe, y compris en anticipant un peu sur l'avenir, en se disant là, il y a quelque chose à creuser, et en essayant de trouver des occasions ou des personnages. Et il faut que ces personnages comprennent le projet, qui est de montrer qui ils sont, sachant qu'on parle rarement d'eux sous cet angle-là, c'est-à-dire celui de la vie réelle. Je pense au travail que j'ai mené avec Nénette, Bonnie, Karim et Pilar dans Images de Marseille, Question de fraternité. II y a un petit livre que j'ai oublié de vous citer, que j'ai publié en 1965, qui s'appelait La photographie, moyen d'expression et instrument de démocratie. J'y exposais une démarche méthodologique pour utiliser la photographie comme moyen d'investigation sur des sujets d'intérêt général, et ce avec la participation directe des gens ou des groupes directement concernés par le sujet. Et ça, aussi bien pendant l'élaboration de l'enquête, que pendant sa réalisation, sa mise en forme (c'est-à-dire la sélection des images ou des sons) et pendant l'exploitation et, si possible, dans un processus itératif, c'est-à-dire avec des feedbacks en plusieurs étapes pour essayer d'approfondir les sujets avec les gens directement concernés.
Pour revenir à votre question, je ne pense pas que je photographie les immigrés différemment d'autres populations ou d'autres sujets. C'est-àdire en y passant du temps, en ne me contentant pas de faire une seule photo mais en travaillant avec des séries d'images, en y revenant plusieurs fois et ensuite en essayant, dans l'editing, de trouver les images les plus fortes.

J'ai parlé des séries. Elles peuvent être composées d'une photo, de deux photos et parfois de 500, comme c'est le cas sur Sarcelles ou sur Fos. II y a des séries thématiques, par exemple la Maison des jeunes et de la culture à Sarcelles, ou bien pour Fos des séries sur différents aspects de la mise en œuvre de ce chantier et des conditions de vie, non seulement des travailleurs mais aussi des habitants autour. Ces séries se constituent, au départ, par des planches contact (je parle de l'époque de l'argentique, mais de toute façon le processus est le même). Un film de 36 vues, $24 \times 36$, revient du labo avec une planche contact sur laquelle on opère une sélection, qu'on appelle l'editing. On sélectionne d'abord des images qui sont exploitables au niveau technique, puis les plus intéressantes du point de vue de l'expression et de l'information. On les repère, avec éventuellement une cote différente pour les meilleures et les moins intéressantes, et on obtient ainsi une présélection. Au verso de la planche contact, on inscrit les légendes scrupuleusement, et ça, pas un mois après, car l'information doit être précise et datée, avec le lieu, etc. Tout cela constitue la matière première d'une collection, qui va permettre une exploitation documentaire : une photographie qui n'est pas légendée ne peut pas mériter le titre de photographie documentaire. Ce n'est pas une information.

Dans une série-sujet, qu'il y ait dix photos ou quinze, on aborde mieux le sujet, plus complètement en tout cas, que s'il n'y avait qu'une photographie. Certaines images ont valeur, je dirais, d'adjectifs voire de virgules, et certaines images 


\section{COLLECTIONS}

sont des verbes, des images plus fortes. Mais les autres viennent les compléter par le moment, par l'angle, et en disent plus sur le sujet que ne pourrait le faire une image isolée. Et puis, parfois, dans une collection, il y a des images qui peuvent mériter plus ou moins le titre sinon d'icônes, d'images emblématiques, qui sont assez souvent demandées pour des livres ou pour la presse, parce que, isolées, elles représentent de manière très forte, symbolique, une thématique.

\section{H\&M : Dans vos écrits, vous évoquez l'importance de " proposer une mémoire visuelle documentaire " mais également l'importance et la difficulté ensuite de faire circuler ces photographies " pour susciter le débat ". Quelle place peut tenir la photographie documentaire dans nos sociétés, notamment par rapport aux questions liées à l'immigration ?}

J. W. : La photographie documentaire peut-elle exister avant de devenir " historique " ? C'est tout le problème. Qu'il s'agisse de mon travail sur Fos ou sur l'immigration. Prenons ce dernier thème puisque c'est lui qui nous réunit. Ce n'est que depuis quelques années que je vois sortir mes photographies sur l'immigration. Sortir dans la presse ou dans des bouquins, faire des couvertures, etc. Mais, dans le temps même où elles ont été faites, mis à part le projet Images de Marseille, Question de fraternité, pendant mon travail sur Fos par exemple, c'était très difficile de les sortir. Parce qu'une bonne photographie documentaire, elle dérange. Elle dérange parce que ce n'est pas une image stéréotypée, c'est-à-dire qui répond à la demande, ou qui est une illustration des représentations déjà enregistrées et connues. Elle peut déranger des pouvoirs, mais aussi des savoirs, y compris auprès des scientifiques. Les gens des sciences humaines avec qui j'ai collaboré sont des gens qui acceptaient un travail pluri voire transdisciplinaire, qui étaient conscients que le réel pouvait être aussi questionné d'une manière différente, plus ouverte, avec le regard d'un photographe.

La photographie documentaire, tout du moins c'est celle qui m'intéresse, est selon moi politique, au sens étymologique du terme "politique " puisqu'elle concerne la gestion de la cité. Je n'ai pas, en disant que je fais de la photographie politique, de posture militante, c'est-à-dire que je n'appartiens pas à un parti, je ne défends pas une cause très étroite. Je m'intéresse à la vie des gens, à la vie des peuples, de telle manière que je puisse, autant que faire se peut, donner la parole aux gens ordinaires.

Mais cette photographie est difficile à produire. II faut trouver des financements (un photographe essaie de manger tous les jours, si possible deux fois par jour, et sa famille aussi, et il y a des coûts techniques, des déplacements, etc.) et, pour susciter le débat, il faut également trouver des commanditaires.

Donc, se pose là la question de la commande publique. Mais une commande publique qui accepte toute la spécificité de la photographie documentaire, qui n'est pas une photographie de communication, ni de propagande, et qui n'est pas produite à l'occasion d'une commémoration ou d'un anniversaire. Mais une photographie qui suppose de la part des financeurs un regard d'investigation. Donc, que l'on mette au jour des questions qui peuvent être gênantes, l'essentiel étant, pour moi, de produire des images qui suscitent le débat, la confrontation. Au sein des institutions, il faut donc trouver des décisionnaires qui acceptent de jouer ce jeu-là, de payer pour cette photographie-là, sachant que cela pourra poser des questions.

Car je pense que la photographie documentaire ne peut exister et être produite que par des 
commandes publiques. La presse ne vous donne pas de temps. Là, le plus souvent, on est obligé de faire la photo attendue et donc on retombe dans l'illustration. Ça me permet de dire à cette occasion que, dans tout mon parcours, c'est surtout avec le secteur institutionnel - public ou parapublic - que j'ai travaillé, davantage qu'avec la presse, parce que seuls des projets financés sur fonds publics ou des commandes publiques m'ont permis de travailler dans la durée.

\section{En savoir plus :}

39 photographies de Jacques Windenberger font partie des collections du Musée national de l'histoire et des cultures de l'immigration. Elles sont consultables en ligne sur le site Internet du musée : http://www.histoire-immigration.fr/ collections/windenberger-jacques

En 2004, Jacques Windenberger a, dans le cadre d'un partenariat avec la Bibliothèque publique d'information du Centre Georges-Pompidou, sélectionné 9 ooo images issues de ses archives (des photographies réalisées entre 1956 et 2002), les a constituées en séries-sujets et les a indexées.

En 2007, il a fait une donation aux Archives départementales des Bouches-du-Rhône de ses archives personnelles (écrits et enregistrements réalisés pendant ses enquêtes) et de ses photographies.

Site Internet du collectif Argos : http://www.collectifargos.com
La grande difficulté aujourd'hui et je terminerai là-dessus, c'est qu'on ne sait plus comment produire et encore moins comment être diffusé. Si j'ai rejoint un collectif, en l'occurrence Argos, c'est aussi pour ne plus être seul sur divers plans de la réflexion, de la critique, etc. Mais c'est aussi une des rares voies aujourd'hui, avec certaines agences qui ressemblent à des collectifs, qui incarne un moyen d'échapper au diktat des grosses agences qui régissent le marché mondial.

\section{Bibliographie de Jacques Windenberger :}

Jacques Windenberger, La photographie, moyen d'expression, instrument de démocratie, Ivry-sur-Seine, Éditions ouvrières, 1965.

Jacques Windenberger, Alain Lebaube, Jean-Pierre Dumont, L'URSS à l'ombre des héros, Paris, éd. Economica, 1980. acques Windenberger, Images de Marseille, Question de fraternité, Paris, éd. Alternatives, 1994.

Jacques Windenberger, Temps de pause, 40 ans de photographie documentaire, Marseille, éd. Bik et Book, 1999.

Jacques Windenberger, Estelle Granet, ONG Solidariedade, Porto Alegre, les voix de la démocratie, Paris, éd. Charles Léopold Mayer/Syllepse.

Jacques Windenberger, Est-ce ainsi que les gens vivent? Chronique documentaire 1969-2002, Marseille, éd. Parenthèse, 2005. Jacques Windenberger, textes de JeanMarie Guillon, Un même monde. Parcours documentaire 1956-2008, Marseille, éd. Images en manœuvres, 2011. 